\section{Prevalência do exame preventivo de câncer do colo do útero em Rio Branco, Acre, Brasil, e fatores associados à não-realização do exame}

\author{
Prevalence of uterine cervical cancer testing in Rio \\ Branco, Acre State, Brazil, and factors associated \\ with non-participation in screening
}

Maria Fernanda de Sousa Oliveira Borges 1 Leila Maria Geromel Dotto 1 Rosalina Jorge Koifman 2 Margarida de Aquino Cunha 1 Pascoal Torres Muniz 1

\section{Introdução}

The objective of the study was to determine the self-reported prevalence of uterine cervical cancer testing in Rio Branco, Acre State, Brazil, and to analyze factors associated with non-participation in screening. A population-based cross-sectional study with a sample of 772 women 18 to 69 years of age and living in Rio Branco was conducted in 2007 and 2008. Data were analyzed with Stata 10.0, and prevalence rates were estimated with Poisson multivariate regression (95\%CI). 85.3\% of women reported at least one cervical cancer screening test in the previous three years. The majority of women (72.8\%) were screened in the Brazilian Unified National Health System. After adjusting for selected variables, statistically significant prevalence rates for absence of screening were found in women 18-24 and 60-69 years of age, single, and with low income and low schooling. The prevalence rates were consistent with findings from other studies, highlighting the need for greater intervention in the group of women most vulnerable to cervical cancer incidence and mortality.

Vaginal Smears; Uterine Cervical Neoplasms; Mass Screening
No Brasil, de acordo com as estimativas do Instituto Nacional de Câncer (INCA) para os anos de 2010 e 2011, excetuando o câncer de pele não melanoma, o câncer do colo do útero foi considerado o segundo tipo de neoplasia mais incidente no sexo feminino, com risco estimado de 18/100 mil mulheres, sendo que na Região Norte este câncer é o mais frequente, apresentando taxas de incidência de 23/100 mil mulheres 1 .

Em relação à mortalidade por câncer do colo do útero, dados oficiais do país apresentam taxa de mortalidade ajustada por idade de 4,9/100 mil mulheres, sendo o Norte a região onde se encontra a mais elevada taxa de mortalidade, 6/100 mil mulheres (Departamento de Informática do SUS. Indicadores e dados básicos - Brasil - 2009. http://tabnet.datasus.gov.br/cgi/idb2009/ma triz.htm, acessado em Fev/2011).

Considerando que esta neoplasia apresenta lesões precursoras com bom prognóstico se diagnosticadas e tratadas precocemente, a forma de abordagem preconizada para o controle populacional consiste na realização do rastreamento através do exame preventivo para câncer do colo do útero 2 . Este também é conhecido como exame de Papanicolaou, um procedimento simples e de baixo custo, capaz de detectar as alterações em fases pré-malignas, quando são curáveis com medidas relativamente simples 3 , reduzindo o risco cumulativo de câncer do colo do útero em 
$84 \%$ para mulheres rastreadas a cada cinco anos, e em $91 \%$ para mulheres que se submetem ao exame a cada três anos 4 .

A Organização Mundial da Saúde (OMS) recomenda a cobertura de $80 \%$ a $85 \%$ de rastreamento da população de risco com o exame Papanicolaou 5. No Brasil, é indicado para mulheres que já tiveram relação sexual, especialmente dos 25 aos 59 anos de idade, com periodicidade anual, sendo trienal quando dois exames anuais seguidos apresentarem resultados negativos para displasia ou neoplasia $6,7,8$.

Apesar das elevadas taxas de morbimortalidade por câncer do colo uterino na Região Norte, observa-se que são escassos os estudos sobre cobertura do exame preventivo nesta região. Sendo assim, o objetivo do presente trabalho consistiu em estimar a cobertura do exame preventivo para câncer do colo do útero no Município de Rio Branco, capital do Acre, Brasil, nos três anos anteriores à pesquisa e avaliar fatores associados à não-realização do exame.

\section{Métodos}

Os dados analisados neste trabalho fazem parte do Inquérito de Saúde e Nutrição de Crianças e Adultos de Rio Branco-Acre, um estudo transversal, de base populacional, realizado no $\mathrm{Mu}$ nicípio de Rio Branco, no qual a amostragem foi obtida por conglomerados em dois estágios de seleção, sendo o primeiro estágio a seleção de setores censitários e o segundo estágio a seleção de domicílios dentro de cada setor, onde foram entrevistados os residentes acima de 18 anos, configurando-se em uma amostra representativa da população geograficamente distribuída no Município de Rio Branco.

No primeiro estágio de seleção, foi utilizada como unidade primária amostral a divisão do Município de Rio Branco em 250 setores censitários, elaborada pelo Instituto Brasileiro de Geografia e Estatística (IBGE; http://www.ibge. gov.br) para o Censo Demográfico do ano 2000, dos quais foram selecionados os 35 setores censitários utilizados anteriormente pela Pesquisa Nacional por Amostra de Domicílios (PNAD) em 2006.

No segundo estágio de seleção, foram sorteados 25 domicílios de cada setor, totalizando 875 domicílios. A fim de suprir prováveis perdas e recusas, foram selecionados $15 \%$ de domicílios a mais, elevando a amostra para 977 domicílios. O cálculo do tamanho amostral esteve dentro dos critérios aceitáveis de precisão com estimativa de 1.500 entrevistas para valores de prevalências para homens e mulheres acima de 18 anos resi- dentes no município, sendo verificadas margens de erro (d) não superiores a 5\% e coeficientes de variação inferiores a $10 \%$, obtidos por meio da fórmula:

$$
C V(p)=\frac{\frac{\sqrt{\mathrm{p}-\mathrm{q}}}{\mathrm{n}}}{\mathrm{p}} ;(\mathrm{d})=(1,96 \mathrm{xepa})
$$

Todos os homens e mulheres, acima de 18 anos, residentes nos domicílios selecionados foram convidados a participar da pesquisa, resultando em uma amostra de 1.516 indivíduos.

A população fonte do inquérito foi composta de 863 mulheres. Para o presente estudo, foram elegíveis mulheres entre 18 e 69 anos de idade, totalizando 817 participantes, sendo registrada uma perda de 5,5\% (45) devido a não resposta do módulo relacionado ao exame preventivo para câncer do colo do útero, o que resultou numa amostra de 772 mulheres.

Para a coleta de dados, foi elaborado um questionário baseado no instrumento utilizado no Inquérito Domiciliar Sobre Comportamentos de Risco e Morbidade Referida de Doenças e Agravos Não Transmissíveis ${ }^{9}$, abordando perguntas distribuídas em quatro seções: (1) sociodemográfica e econômica; (2) avaliação do uso e cobertura de serviços de saúde; (3) avaliação do estado de saúde individual, com dados de morbidade referida, estilo de vida, atividade física, entre outros e; (4) avaliação do estado nutricional. No bloco de avaliação do estado de saúde individual, havia o módulo sobre exame preventivo de câncer do colo do útero. Os dados foram coletados no período de novembro de 2007 a outubro de 2008, através de entrevista realizada por uma equipe de auxiliares de pesquisa treinados para aplicação do instrumento.

A prevalência de realização do exame preventivo para câncer de colo do útero foi definida como ter realizado o exame nos três anos anteriores à data da entrevista. Dentre as mulheres que se submeteram ao exame, foi avaliado o relato de frequência, local da demanda do último exame (rede pública ou privada) e a indicação para sua realização.

As variáveis independentes estudadas nos aspectos sociodemográficos foram idade (em anos) na data da investigação, situação conjugal, escolaridade, renda (em salários mínimos) e trabalho fora de casa. As variáveis relacionadas ao estilo de vida e condições de saúde incluíram o habito do tabagismo (indivíduos que se denominavam fumantes no momento da entrevista), autopercepção de saúde, padrão de alimentação, prática de atividade física, submissão à dosagem de glicose e colesterol sanguíneos (utilizados como aproximações indiretas do 
uso dos serviços de saúde), presença de alguma morbidade autorreferida, diabetes e hipertensão autorreferidas.

Para avaliar o hábito alimentar, foi criado um “índice de alimentação saudável”. No questionário as variáveis referentes à alimentação foram coletadas por meio de escala de frequência de consumo alimentar ( 1 a 2 dias por semana; 3 a 4 dias por semana; 5 a 6 dias por semana; todos os dias; quase nunca ou nunca). Estas foram transformadas em dicotômicas: consumo usual (todos os dias/de 1 a 6 dias por semana) e consumo não usual (quase nunca/nunca). Os itens contidos no questionário foram classificados como marcadores de consumo alimentar saudável (peixe, feijão, frutas, legume ou vegetal cozido, gordura vegetal, salada crua) e marcadores de consumo alimentar menos saudável (leite integral, carne vermelha com gordura, frango com pele, refrigerante). Estas variáveis foram dicotomizadas em consumo usual sim ou não e codificadas como 0 para sim e 1 para não, nas variáveis marcadoras de consumo alimentar saudável, e 0 para não e 1 para sim nas variáveis marcadoras de consumo alimentar menos saudável. A soma total dessas variáveis é igual a 10. Definiu-se o valor 5 como ponto de corte, onde consideramos a pontuação de 0 a 4 como hábito alimentar mais saudável e de 5 a 10 hábito alimentar menos saudável. Construiu-se, assim, a variável dicotômica alimentação saudável 10 .

A variável prática de atividade física foi elaborada tendo como referência a classificação da OMS 11 sobre prática de atividade física ocupacional e de lazer. Considerou-se prática de atividade física ocupacional quando o indivíduo respondeu "sim" para pelo menos uma das questões: "No seu trabalho, anda bastante a pé?"; "No seu trabalho, carrega peso ou faz outra atividade pesada?"; "Costuma ir a pé ou de bicicleta de casa para o trabalho?"; "Fica com a parte mais pesada da faxina?”. Já a prática de atividade física de lazer foi classificada segundo afirmação do entrevistado ao responder se faz algum tipo de exercício físico. Portanto, a variável dicotômica atividade física foi criada considerando as respostas das duas variáveis supracitadas.

O banco de dados foi construído utilizando o pacote estatístico Epi Info 6.0 (Centers for Disease Control and Prevention, Atlanta, Estados Unidos) e para análise dos dados foi utilizado o programa Stata 10.0 (Stata Corp., College Station, Estados Unidos), por meio do qual as variáveis de interesse do estudo foram submetidas à análise descritiva, sendo determinadas medidas de associação através das razões de prevalências (RP). Foi utilizado o teste de qui-quadrado de Pearson e estimadas as RP a partir da regressão multivaria- da de Poisson, considerando-se estatisticamente significativos os valores de $\mathrm{p}<0,05$ (intervalo de 95\% de confiança - IC95\%) e como desfecho dessa análise a não realização do exame nos últimos três anos, a fim de identificar as características associadas com a ausência de adesão ao exame na população estudada.

O modelo de regressão de Poisson foi construído com a entrada das variáveis com valor de $\mathrm{p}<0,20$ na analise bivariada, permanecendo no modelo final as variáveis com valor de $\mathrm{p}<0,05$. As variáveis foram adicionadas passo a passo no modelo, sendo incluídas inicialmente as variáveis sociodemográficas seguidas das variáveis relacionadas ao estilo de vida e condições de saúde.

A análise foi ponderada, utilizando-se comandos do módulo svy (survey comands) do pacote estatístico Stata, o qual toma em consideração os pesos, estratos e unidades primárias de amostragem.

Em cumprimento aos requisitos exigidos pela Resolução $n^{o}$. 196/96 do Conselho Nacional de Saúde, o projeto foi submetido à apreciação e aprovado pelo Comitê de Ética em Pesquisa da Universidade Federal do Acre. Todos os entrevistados foram informados quanto à participação voluntária na pesquisa, sendo-lhes assegurado o sigilo das informações por meio do Termo de Consentimento Livre e Esclarecido assinado pelos participantes.

\section{Resultados}

$\mathrm{Na}$ amostra elegível para esta pesquisa (772 mulheres), a média de idade foi de 36,6 anos (mediana 34 anos, primeiro quartil 25 anos, e terceiro quartil 46 anos), a maioria era casada ou com união consensual $(55,3 \%)$, possuía até o Ensino Fundamental completo $(65,3 \%)$, não trabalhava fora de casa $(56,5 \%)$, apresentava renda de até um salário mínimo (54,4\%), já havia se submetido a exame de colesterol sanguíneo $(60,6 \%)$ e exame de glicose sanguínea alguma vez na vida $(57,1 \%)$.

O percentual de mulheres que mencionaram haver realizado o exame de Papanicolaou pelo menos uma vez nos três anos anteriores, foi de 75,3\% (586 mulheres). Considerando somente a população-alvo para a realização do exame, constituída por mulheres de 25 a 59 anos, conforme preconizado pelo Ministério da Saúde, o percentual de adesão ao exame se eleva para 85,3\% (453 mulheres) (Tabela 1).

A maioria das mulheres que se submeteu ao exame $(75 \%)$ utilizou a rede do Sistema Único de Saúde (SUS) como local para a última coleta, 
$84,7 \%$ afirmaram sua realização como exame de rotina, e $61,7 \%$ referiram adotar uma periodicidade anual (Tabela 2).

Apresentaram maiores prevalências para realização do exame mulheres que pertenciam à faixa etária de 25 e 35 anos $(86,4 \%)$, seguida da faixa etária de 35 a 50 anos $(85,9 \%)$, casadas ou com união estável (83,6\%), que possuíam o Ensino Superior ou mais $(80,4 \%)$, trabalhavam fora de casa $(82,5 \%)$ e informavam renda acima de 2 salários mínimos (83,7\%). Quanto às características de estilo de vida e condições de saúde, maiores prevalências de adesão ao exame preventivo foram encontradas entre aquelas que praticavam atividade física $(82,2 \%)$ e haviam realizado exame de colesterol $(83,1 \%)$ e glicose sanguínea $(82,6 \%)$, resultados com significância estatística (Tabela 3).

\section{Tabela 1}

Distribuição de frequências de relatos autorreferidos quanto à realização do exame Papanicolaou nos últimos três anos, segundo idade, amostra de mulheres de base populacional. Rio Branco, Acre, Brasil, 2007-2008.

\begin{tabular}{|c|c|c|c|}
\hline $\begin{array}{l}\text { Relato de realização de } \\
\text { exame Papanicolaou }\end{array}$ & $\begin{array}{l}\text { Mulheres elegíveis do } \\
\text { inquérito (18-69 anos) }\end{array}$ & $\begin{array}{l}\text { Mulheres-alvo do } \\
\text { programa-Ministério da } \\
\text { Saúde (25-59 anos) }\end{array}$ & $\begin{array}{c}\text { Mulheres }<25 e \\
>59 \text { anos }\end{array}$ \\
\hline & n (\%) & n (\%) & n (\%) \\
\hline $\operatorname{Sim}(\%)$ & $586(75,3)$ & $453(85,3)$ & $133(54,3)$ \\
\hline Não (\%) & $186(24,7)$ & $79(14,7)$ & $107(45,7)$ \\
\hline Total & $772(100,0)$ & $532(100,0)$ & $240(100,0)$ \\
\hline
\end{tabular}

Tabela 2

Distribuição de frequências de relatos autorreferidos quanto à realização do exame Papanicolaou nos últimos três anos segundo local do exame, motivo e frequência de realização, amostra de mulheres de base populacional. Rio Branco, Acre, Brasil, 2007-2008.

\begin{tabular}{|c|c|c|c|c|c|c|}
\hline \multirow[t]{2}{*}{ Variáveis } & \multicolumn{2}{|c|}{$\begin{array}{l}\text { Mulheres de 18-69 anos } \\
\qquad(n=586) \text { * }\end{array}$} & \multicolumn{2}{|c|}{$\begin{array}{l}\text { Mulheres de } 25-59 \text { anos } \\
\qquad(n=532) \text { * }\end{array}$} & \multicolumn{2}{|c|}{$\begin{array}{c}\text { Mulheres }<25 \text { e }>59 \text { anos } \\
(n=240) \text { * }\end{array}$} \\
\hline & n (\%) & Valor de $p$ & n (\%) & Valor de $p$ & n (\%) & Valor de $p$ \\
\hline \multicolumn{7}{|c|}{ Local de realização do exame } \\
\hline Rede SUS & $417(75,0)$ & $<0,001$ & $329(72,8)$ & $<0,001$ & $88(66,7)$ & $<0,001$ \\
\hline Rede privada & $167(25,0)$ & & $123(27,2)$ & & $44(33,3)$ & \\
\hline \multicolumn{7}{|l|}{ Motivo para realização do } \\
\hline \multicolumn{7}{|l|}{ exame } \\
\hline Exame de rotina & $511(84,7)$ & $<0,001$ & $393(87,0)$ & $<0,001$ & $119(89,5)$ & $<0,001$ \\
\hline Problemas anteriores & $71(15,0)$ & & $57(12,6)$ & & $14(10,5)$ & \\
\hline Outros & $2(0,3)$ & & $2(0,4)$ & & & \\
\hline \multicolumn{7}{|l|}{ Frequência de realização } \\
\hline \multicolumn{7}{|l|}{ do exame } \\
\hline Só fez uma vez & $30(5,1)$ & $<0,001$ & $18(4,1)$ & $<0,001$ & $12(9,6)$ & $<0,001$ \\
\hline Semestral & $86(15,1)$ & & $72(16,3)$ & & $14(11,2)$ & \\
\hline Anual & $350(61,7)$ & & $278(62,4)$ & & $72(57,6)$ & \\
\hline A cada 2 anos & $40(7,1)$ & & $27(6,1)$ & & $13(10,4)$ & \\
\hline A cada 3 anos & $10(1,8)$ & & $7(1,6)$ & & $3(2,4)$ & \\
\hline Mais de 3 anos & $12(2,3)$ & & $10(2,7)$ & & $2(1,6)$ & \\
\hline Variável & $39(6,9)$ & & $30(6,8)$ & & $9(7,2)$ & \\
\hline
\end{tabular}

* As diferenças nos valores absolutos das frequências correspondem às perdas. 
Distribuição de frequências de relatos autorreferidos quanto à realização do exame Papanicolaou nos últimos três anos, segundo variáveis socioeconômicas, demográficas, estilo de vida e condições de saúde, amostra de mulheres de base populacional. Rio Branco, Acre, Brasil, 2007-2008.

\begin{tabular}{|c|c|c|c|c|}
\hline \multirow[t]{3}{*}{ Variáveis } & \multirow{3}{*}{$\begin{array}{c}\text { Total }(\mathrm{N}=772) \text { * } \\
\mathrm{n}(\%)\end{array}$} & \multicolumn{2}{|c|}{ Realização do exame Papanicolaou } & \multirow[t]{3}{*}{ Valor de $p$} \\
\hline & & Não $(n=186)$ * & $\operatorname{Sim}(n=586)$ * & \\
\hline & & $n(\%)$ & $n(\%)$ & \\
\hline \multicolumn{5}{|l|}{ Faixa etária (anos) } \\
\hline $18 \mid-25$ & $187(24,2)$ & $87(46,5)$ & $100(53,5)$ & \multirow[t]{4}{*}{$<0,001$} \\
\hline $25 \mid-35$ & $206(26,7)$ & $28(13,6)$ & $178(86,4)$ & \\
\hline $35 \mid-50$ & $220(28,5)$ & $31(14,1)$ & $189(85,9)$ & \\
\hline $50 \mid-69$ & $159(20,6)$ & $40(25,2)$ & $119(74,8)$ & \\
\hline \multicolumn{5}{|l|}{ Status conjugal } \\
\hline Solteira/Divorciada/Viúva & $370(47,9)$ & $120(32,4)$ & $250(67,6)$ & \multirow[t]{2}{*}{$<0,001$} \\
\hline Casado/União estável & $402(52,1)$ & $66(16,4)$ & $336(83,6)$ & \\
\hline \multicolumn{5}{|l|}{ Escolaridade } \\
\hline Não alfabetizado & $29(3,8)$ & $11(37,9)$ & $18(62,1)$ & \multirow[t]{5}{*}{0,356} \\
\hline Alfabetizado & $16(2,1)$ & $6(37,5)$ & $10(62,5)$ & \\
\hline Ensino Fundamental & $281(36,5)$ & $65(23,1)$ & $216(76,9)$ & \\
\hline Ensino Médio & $308(40,1)$ & $76(24,7)$ & $232(75,3)$ & \\
\hline Ensino Superior e mais & $135(17,5)$ & $26(19,3)$ & $109(80,7)$ & \\
\hline \multicolumn{5}{|l|}{ Trabalho } \\
\hline Sim & $365(47,3)$ & $64(17,5)$ & $301(82,5)$ & \multirow[t]{2}{*}{0,003} \\
\hline Não & $407(52,7)$ & $122(30,0)$ & $285(70,0)$ & \\
\hline \multicolumn{5}{|l|}{ Renda (salários minimos) } \\
\hline Sem renda & $299(39,3)$ & $92(30,8)$ & $207(69,2)$ & \multirow[t]{3}{*}{0,001} \\
\hline Menos de 1 e 2 & $296(38,9)$ & $64(21,6)$ & $232(78,4)$ & \\
\hline Acima de 2 & $166(21,8)$ & $27(16,3)$ & $139(83,7)$ & \\
\hline \multicolumn{5}{|l|}{ Fuma } \\
\hline Sim & $107(13,9)$ & $22(20,6)$ & $85(79,4)$ & \multirow[t]{2}{*}{0,873} \\
\hline Não & $665(86,1)$ & $164(24,7)$ & $501(75,3)$ & \\
\hline \multicolumn{5}{|l|}{ Autopercepção de saúde } \\
\hline Excelente & $64(8,3)$ & $15(23,4)$ & $49(76,6)$ & \multirow[t]{4}{*}{0,872} \\
\hline Boa & $346(44,9)$ & $83(24,0)$ & $263(76,0)$ & \\
\hline Regular & $309(40,1)$ & $72(23,3)$ & $237(76,7)$ & \\
\hline Ruim & $52(6,7)$ & $16(30,8)$ & $36(69,2)$ & \\
\hline \multicolumn{5}{|l|}{ Alimentação saudável } \\
\hline Sim & $416(53,9)$ & $83(19,9)$ & $333(80,1)$ & \multirow[t]{2}{*}{0,244} \\
\hline Não & $356(46,1)$ & $103(28,9)$ & $253(71,1)$ & \\
\hline Atividade física & & & & \\
\hline Sim & $152(19,7)$ & $27(17,8)$ & $125(82,2)$ & 0,036 \\
\hline Não & $620(80,3)$ & $159(25,7)$ & $461(74,3)$ & \\
\hline Dosagem de colesterol sang & & & & \\
\hline $\operatorname{Sim}$ & $478(62,1)$ & $81(16,9)$ & $397(83,1)$ & $<0,001$ \\
\hline Não & $292(37,9)$ & $104(35,6)$ & $188(64,4)$ & \\
\hline Dosagem de glicose sanguín & & & & \\
\hline $\operatorname{Sim}$ & $448(58,0)$ & $78(17,4)$ & $370(82,6)$ & $<0,001$ \\
\hline Não & $324(42,0)$ & $108(33,3)$ & $216(66,7)$ & \\
\hline Morbidade autorreferida & & & & \\
\hline Sim & $179(23,2)$ & $54(30,2)$ & $125(69,8)$ & 0,07 \\
\hline Não & $593(76,8)$ & $132(22,3)$ & $461(77,7)$ & \\
\hline Hipertensão autorreferida & & & & \\
\hline Sim & $212(27,5)$ & $37(17,5)$ & $175(82,5)$ & 0,229 \\
\hline Não & $560(72,5)$ & $149(26,6)$ & $411(73,4)$ & \\
\hline Diabetes autorreferida & & & & \\
\hline $\operatorname{Sim}$ & $29(3,8)$ & $4(13,8)$ & $25(86,2)$ & 0,176 \\
\hline Não & $743(96,2)$ & $182(24,5)$ & $561(75,5)$ & \\
\hline
\end{tabular}

* As diferenças nos valores absolutos das frequências correspondem às perdas. 
Quanto à análise da regressão multivariada de Poisson, para verificar fatores associados com a não realização do exame entre as mulheres de Rio Branco, as RP brutas mostraram que o estrato de mulheres não pertencentes à faixa etária alvo do programa do Ministério da Saúde, sem união estável, que não trabalhavam fora de casa e com menor renda apresentaram maiores estimativas de risco para não realização do exame.

Após ajuste com as variáveis idade, estado civil, renda e escolaridade, a variável trabalho externo ao domicílio perdeu significância estatística, e a variável escolaridade passou a apresentar associação com a não realização do exame, expressando RP de 1,74 (IC95\%: 1,37-2,20) entre mulheres com escolaridade até o ensino fundamental comparativamente com aquelas de maior escolaridade.

$\mathrm{Na}$ análise ajustada, a faixa etária de menos de 25 anos apresentou RP de 3,19 (IC95\%: 2,23$4,57)$ para não realização do exame comparativamente com a faixa etária alvo. Na população acima de 59 anos, a magnitude de associação foi de 2,05 (IC95\%: 1,45-2,88) (Tabela 4).

Em relação à análise de associação entre as características de estilo de vida e saúde autorreferida com a não adesão ao Papanicolaou, foi observada associação somente com a variável não realização de dosagem de colesterol $(\mathrm{RP}=1,65$; IC95\%: 1,12-2,42), após ajuste com idade, estado civil, renda e escolaridade (Tabela 5).

\section{Discussão}

O percentual de cobertura do exame preventivo para câncer do colo do útero encontrado neste estudo (85,3\% na faixa etária de 25 a 59 anos) revela boa adesão ao exame de Papanicolaou pelas mulheres da capital do Acre, alcançando os padrões recomendados para a população de risco pela OMS e pelo Programa Nacional de Controle do Câncer do Colo do Útero e Mama do Ministério da Saúde do Brasil 5,6. Em relação a essa população alvo, o percentual de adesão ao exame de Papanicolaou de Rio Branco equipara-se aos melhores resultados de inquéritos semelhantes realizados em outras capitais brasileiras 9 .

Uma revisão sistemática de literatura apontou que até o ano de 2004 foram realizados no Brasil 13 estudos transversais sobre cobertura do exame de Papanicolaou, sendo quatro com amostragem não aleatória e nove de base populacional. Destes, quatro foram realizados em São Paulo (1987, 1988, 2000 e 2002), quatro no Rio Grande do Sul (1995, 1999, 2000 e 2002) e um no

Tabela 4

Razão de prevalências (RP) de relatos autorreferidos de ausência na realização do exame Papanicolaou nos últimos três anos, segundo variáveis sociodemográficas selecionadas, amostra de mulheres de base populacional. Rio Branco, Acre, Brasil, 2007-2008.

\begin{tabular}{|c|c|c|}
\hline Variáveis & RP bruta (IC95\%) & RP ajustada * (IC95\%) \\
\hline \multicolumn{3}{|l|}{ Idade (anos) } \\
\hline 18|-25 & $3,25(2,24-4,74)$ & $3,19(2,23-4,57)$ \\
\hline $25 \mid-60$ & 1,00 & 1,00 \\
\hline $60 \mid-69$ & $2,65(1,97-3,56)$ & $2,05(1,45-2,88)$ \\
\hline \multicolumn{3}{|l|}{ Escolaridade (anos) } \\
\hline 8 e mais & 1,00 & 1,00 \\
\hline $0-7$ & $1,36(0,99-1,86)$ & $1,74(1,37-2,20)$ \\
\hline \multicolumn{3}{|l|}{ Estado civil } \\
\hline Casada/União estável & 1,00 & 1,00 \\
\hline Solteira/Divorciada/Viúva & $1,77(1,20-2,60)$ & $1,77(1,22-2,58)$ \\
\hline \multicolumn{3}{|l|}{ Trabalho } \\
\hline Sim & 1,00 & 1,00 \\
\hline Não & $1,67(1,20-2,24)$ & $1,09(0,69-1,74)$ \\
\hline \multicolumn{3}{|l|}{ Renda (salários mínimos) } \\
\hline Maior que 1 & 1,00 & 1,00 \\
\hline Sem renda ou menor que 1 & $1,59(1,30-1,93)$ & $1,47(1,13-1,92)$ \\
\hline
\end{tabular}

* Ajustada para idade, escolaridade, estado civil, trabalho e renda. 
Razão de prevalências (RP) de relatos autorreferidos de ausência na realização do exame Papanicolaou nos últimos três anos, segundo percepção de saúde, hábitos de vida e consumo de serviços de saúde, amostra de mulheres de base populacional. Rio Branco, Acre, Brasil, 2007-2008.

\begin{tabular}{|c|c|c|}
\hline Variáveis & RP bruta (IC95\%) & RP ajustada * (IC95\%) \\
\hline \multicolumn{3}{|c|}{ Alimentação saudável } \\
\hline Sim & 1,00 & 1,00 \\
\hline Não & $1,20(0,83-1,67)$ & $1,16(0,87-1,54)$ \\
\hline \multicolumn{3}{|l|}{ Atividade física } \\
\hline Sim & 1,00 & 1,00 \\
\hline Não & $1,52(1,01-2,29)$ & $1,21(0,76-1,91)$ \\
\hline \multicolumn{3}{|c|}{ Dosagem de colesterol sanguíneo } \\
\hline Sim & 1,00 & 1,00 \\
\hline Não & $2,00(1,52-2,63)$ & $1,65(1,12-2,42)$ \\
\hline \multicolumn{3}{|c|}{ Dosagem de glicose sanguínea } \\
\hline Sim & 1,00 & 1,00 \\
\hline Não & $1,72(1,27-2,34)$ & $1,04(0,73-1,48)$ \\
\hline \multicolumn{3}{|c|}{ Autopercepção de saúde } \\
\hline Satisfatória & 1,00 & 1,00 \\
\hline Não satisfatória & $0,96(0,69-1,35)$ & $1,02(0,77-1,35)$ \\
\hline \multicolumn{3}{|c|}{ Morbidade autorreferida } \\
\hline Não & 1,00 & 1,00 \\
\hline Sim & $0,69(0,47-1,02)$ & $0,94(0,63-1,41)$ \\
\hline \multicolumn{3}{|c|}{ Hipertensão autorreferida } \\
\hline Não & 1,00 & 1,00 \\
\hline Sim & $0,80(0,55-1,16)$ & $0,88(0,66-1,16)$ \\
\hline \multicolumn{3}{|c|}{ Diabetes autorreferida } \\
\hline Não & 1,00 & 1,00 \\
\hline Sim & $0,52(0,18-1,48)$ & $0,69(0,15-3,12)$ \\
\hline \multicolumn{3}{|l|}{ Tabagismo } \\
\hline Não & 1,00 & 1,00 \\
\hline Sim & $1,02(0,72-1,45)$ & $0,85(0,64-1,13)$ \\
\hline
\end{tabular}

* Razão de prevalência ajustada para idade, estado civil, renda, escolaridade.

Paraná em 2002, com diferenças metodológicas entre estes estudos 12 .

$\mathrm{O}$ inquérito de base populacional realizado em São Paulo, no ano de 2000, composto por uma amostra de 1.172 mulheres com idade entre 15 e 49 anos com atividade sexual, revelou que $77,3 \%$ se submeteram ao exame nos três anos anteriores à pesquisa 13. Já no inquérito de Pelotas (Rio Grande do Sul), realizado em 2002, foi encontrado o percentual de $68,8 \%$ em uma amostra de 1.198 mulheres de 25 a 59 anos 14 .

Outras pesquisas de base populacional realizadas, como o estudo transversal em São Leopoldo (Rio Grande do Sul) no ano de 2003 revelou uma cobertura de $85,5 \%$ nos últimos três anos 7, um inquérito em Londrina (Paraná), no ano de 2004, encontrou cobertura de $80,7 \% 15$ e em São Luís (Maranhão) 82,4\% das mulheres informaram ter se submetido ao exame pelo menos uma vez 16.

Em 2003, foram realizados três inquéritos de base populacional de abrangência nacional. Um deles foi a Pesquisa Mundial de Saúde conduzida pela OMS em 188 municípios, que identificou uma cobertura de $66 \%$ de realização do exame nos três anos anteriores à entrevista entre mulheres de 18 a 69 anos, porém a pesquisa não contemplou o estado do Acre 17. Outro estudo foi a PNAD, na qual $68,7 \%$ das mulheres acima de 25 anos informaram ter realizado o $\mathrm{Pa}$ panicolaou nos três anos anteriores 18. Já pelo Inquérito Domiciliar sobre Comportamentos de Risco e Morbidade Referida de Doenças e Agravos Não-transmissíveis, realizado entre mulheres de 25 a 59 anos em 15 capitais brasileiras e no Distrito Federal, não incluindo a capital do Acre, a 
cobertura dos exames de Papanicolaou variou de $73,4 \%$ em João Pessoa (Paraíba) a 92,9\% em Vitória (Espírito Santo) ${ }^{9}$.

Comparando nossos achados com os resultados apresentados neste último inquérito ${ }^{9}$, o qual possui aspectos metodológicos similares ao presente estudo, a capital acreana ocupou o sexto lugar de maior adesão ao exame em relação às demais capitais brasileiras, e a primeira posição em relação às capitais selecionadas da Região Norte que participaram da referida pesquisa.

O percentual elevado encontrado em Rio Branco pode estar relacionado à natureza do estabelecimento onde ocorreu o exame, que constatou ser a rede do SUS o principal local de coleta $(75 \%)$, revelando a importância do acesso ao exame de Papanicolaou pelos serviços públicos de saúde nesta capital. Em outras capitais da Região Norte, como Manaus (Amazonas - 63,4\%) e Belém (Pará - 53,7\%), também foram encontrados maiores percentuais de realização do exame no SUS, padrão distinto daquele observado nas regiões Sul e Sudeste, onde a rede privada apresentou maior demanda 9 .

A cobertura do exame preventivo para câncer do colo do útero aumentou entre 2003 e 2008 no país, de acordo com os resultados obtidos pela PNAD, variando de $82,6 \%$ em 2003 para $87,1 \%$ em 2008, no percentual de mulheres na faixa de 25 a 59 anos que haviam realizado alguma vez o exame 18,19. A PNAD no Estado do Acre, cuja amostragem foi composta por municípios selecionados, também apresentou acréscimo percentual, variando de 70,2\% em 2003 para 77,7\% em 2008 na faixa etária mencionada, sendo esses valores relacionados à submissão ao exame nos três anos anteriores à pesquisa (Departamento de Informatica do SUS. Indicadores de cobertura. http:// tabnet.datasus.gov.br/cgi/tabnet.exe?idb2009/ f22.def, acessado em Fev/2011 / IBGE. Estados@. Acre. http://www.ibge.gov.br/estadosat/temas. php?sigla=ac\&tema=pnad_saude_2008, acessado em Fev/2011).

Quanto à periodicidade do exame, a OMS recomenda a realização anual a partir dos 25 aos 59 anos de idade e, caso os resultados sejam negativos para displasia ou neoplasia por dois anos consecutivos, o intervalo poderá ser estendido para três anos 20 . No presente estudo, em torno de $60 \%$ das mulheres relataram uma frequência anual de realização do exame, sugerindo facilidade de acesso aos serviços públicos em Rio Branco. Contudo, neste inquérito, não foi possível estabelecer o vínculo entre o último exame e os resultados de exames anteriores que possibilitassem avaliar a adesão à mencionada recomendação.
Ao analisar os dados desta investigação, é necessário considerar algumas limitações que podem interferir nos resultados de estudos transversais, como vieses de memória e de informação, já que se trata de informações baseadas em autorrelato. Nesse sentido, pode ocorrer o efeito de superestimação da frequência dos exames e subestimação do tempo decorrido em relação ao exame mais recente. Contudo, os estudos transversais são importantes por fornecerem estimativas que auxiliam no planejamento de saúde, e têm sido utilizados para a avaliação da cobertura do exame de Papanicolaou como um procedimento válido em diversas pesquisas 12,13,17.

A faixa etária prioritária estabelecida pelo Programa Nacional de Controle do Câncer do Colo do Útero e Mama do Ministério da Saúde (25 a 59 anos) foi a que mais realizou o exame, reforçando a importância da acessibilidade a este. Ainda que mulheres pertencentes a outras faixas etárias com vida sexual ativa possam ser atendidas pelo programa de prevenção, observa-se menor adesão ao exame tanto entre as mais jovens como entre aquelas de maior idade que a faixa etária estabelecida como alvo ${ }^{12}$. No presente estudo, a menor prevalência de realização do exame foi encontrada dentro do grupo de mulheres com idade inferior a 25 anos.

Pesquisa avaliando a faixa etária de rastreamento em mulheres atendidas nas unidades de saúde de São Paulo identificou que 24,3\% dos casos de lesão intraepitelial escamosa de alto grau, diagnosticados em 2006, ocorreram em mulheres com idade inferior ou igual a 25 anos, com destaque para as adolescentes, sugerindo a necessidade de avaliação das alterações cervicais entre mulheres mais jovens, com ações educativas e preventivas voltadas para esse grupo populacional 21 .

No Estado do Acre, um estudo transversal realizado em 2001 apontou que entre as mulheres na faixa etária de 15 a 19 anos a frequência de lesões precursoras do câncer do colo do útero foi de $6,4 \%$, evidenciando exposição precoce aos fatores de risco para o desenvolvimento de câncer do colo do útero ${ }^{22}$. A associação entre exposição precoce e ausência de rastreamento oportuno pode ocasionar lesões cervicais graves até a idade sugerida para o rastreamento 21 , ressaltando a importância de ações educativas quanto à prevenção e aos fatores de risco para o desenvolvimento das neoplasias do útero.

O principal fator de risco associado com a ocorrência de lesões precursoras e câncer do colo do útero é a infecção pelo papilomavírus humano (HPV), sendo também considerados fatores de risco associados o início precoce de atividades sexuais e a multiplicidade de parceiros sexuais 
masculinos. Outros fatores de risco incluem condições de imunossupressão, fatores nutricionais, história familiar, infecções genitais e uso prolongado de contraceptivos orais embora estejam pouco esclarecidos 23,24,25.

Em relação às mulheres entrevistadas acima de 59 anos, também foi identificada menor adesão ao exame que a observada na faixa etária de 25 a 59 anos. O término da idade fértil parece resultar numa diminuição na realização de consultas ginecológicas, levando ao afastamento das práticas de prevenção em um período do ciclo de vida quando a incidência e gravidade das neoplasias são mais elevadas. No entanto, essa população demanda outros serviços de saúde que poderiam ser aproveitados para a condução da realização do Papanicolaou sob uma visão de integralidade da assistência 26.

Entre os fatores que se associaram à não-realização do exame em Rio Branco, além da variável idade, observou-se que o estado civil, a renda e a escolaridade foram variáveis associadas a este desfecho. Os estratos de mulheres solteiras, com escolaridade até Ensino Fundamental incompleto, e renda inferior a um salário mínimo, apresentaram magnitudes de associação positivas com a não adesão ao exame de Papanicolaou, resultados consistentes com os achados de estudos em outras localidades 7,27,28.

A situação conjugal já foi relatada na literatura como fator associado. Pesquisa realizada entre mulheres hispânicas identificou que o risco para não realizar o exame entre mulheres não casadas ou sem união estável era cerca de quatro vezes maior em relação às casadas 29. De modo semelhante, no estudo em Pelotas, foi observado que ser viúva ou solteira consistia em fator de risco para não realização do exame 14 . Uma possível explicação para esse aspecto indica que mulheres casadas ou em união estável podem estar mais propensas a procurar serviços de planejamento familiar ou obstétricos, oportunizando a realização do exame ${ }^{30}$.

Quanto aos fatores socioeconômicos, estes têm sido apontados como um dos elementos mais importantes relacionados ao comportamento preventivo entre as mulheres, onde baixos níveis de escolaridade e renda estão associados à ausência de rastreamento do câncer do colo do útero, como indicaram diversas pesquisas realizadas no país $14,15,31,32,33$. Na presente investigação, a associação estatisticamente significativa destes fatores com a não realização do preventivo aponta falta de equidade social no acesso ao exame de citologia oncótica. Nesse sentido, baixos níveis socioeconômicos também têm sido associados ao desenvolvimento do câncer do colo do útero, uma vez que populações mais vulneráveis são as que apresentam maiores barreiras de acesso à detecção e tratamento precoces, gerando a necessidade de maior intervenção direcionada a estes segmentos, que são os que mais dependem dos serviços públicos de saúde 33 .

Sendo assim, faz-se necessário incentivar o rastreamento do câncer de colo do útero no grupo específico de mulheres, cujas estimativas de risco estiveram positivamente associadas à não-realização do exame, composto, sobretudo, pelos estratos de mulheres não pertencentes à faixa etária prioritária do programa, solteiras, com menor renda e baixa escolaridade.

Quanto às variáveis relacionadas ao autocuidado e à percepção autorreferida de saúde, foi encontrada associação para não realização do exame de Papanicolaou somente naquelas mulheres que não haviam realizado dosagens dos níveis séricos de colesterol, como também relatado em estudo realizado no Rio de Janeiro e Fortaleza (Ceará) 31.

Entre as limitações desta investigação, podese mencionar a ausência de exploração de alguns fatores de risco para câncer do colo uterino, não obtidos na realização do inquérito, como o início precoce da atividade sexual, a multiplicidade de parceiros sexuais, entre outros, impossibilitando essa análise.

Entre os pontos fortes, destaca-se a obtenção pioneira de estimativas de base populacional da prevalência e fatores associados à não-realização do exame no Município de Rio Branco, podendo contribuir para o planejamento das atividades oferecidas pelo setor público à saúde da mulher. Inquéritos de base populacional constituem-se em importantes instrumentos para gerar informações que possibilitem o conhecimento das condições de vida e saúde das populações, sendo realizado de forma crescente e utilizado como elemento essencial dos sistemas de informação para o planejamento e a avaliação dos serviços de saúde. Além disso, os inquéritos possibilitam a aquisição de dados de indivíduos que não utilizam os serviços, permitindo a superação de uma grande limitação oriunda de dados obtidos a partir da demanda ou de registros de atendimentos das instituições de saúde, o que favorece a avaliação de grupos com diferentes características socioeconômicas e demográficas 34 .

Considerando os elevados índices de morbimortalidade por câncer do colo do útero na região, faz-se necessário em futuras investigações analisar a qualidade dos exames citopatológicos realizados pelos serviços públicos de saúde e o seguimento das mulheres submetidas ao teste, a fim de garantir a eficiência da cobertura populacional do exame preventivo. 


\section{Resumo}

O objetivo do estudo foi determinar a prevalência autorreferida do exame preventivo de câncer do colo uterino em Rio Branco, Acre, Brasil, e avaliar fatores associados com a não realização do exame. Trata-se de estudo transversal de base populacional, composto por 772 mulheres de 18 a 69 anos residentes em Rio Branco, no período 2007 a 2008. Os dados foram analisados no programa Stata 10.0 e estimadas razões de prevalências a partir da regressão multivariada de Poisson. O percentual de mulheres na população alvo que relatou pelo menos um exame nos últimos três anos foi de 85,3\%, com acentuada utilização do Sistema Único de Saúde (72,8\%). Foram encontradas razões de prevalência estatisticamente significativas quanto à ausência do exame em mulheres de 18 a 24 anos, de 60 a 69 anos, solteiras, com menor renda e baixa escolaridade. As magnitudes encontradas nas razões de prevalência foram consistentes com achados de outros estudos, apontando maior necessidade de intervenção no grupo de mulheres mais vulneráveis à incidência e mortalidade por câncer do colo do útero.

Esfregaço Vaginal; Neoplasias do Colo do Útero; Programas de Rastreamento

\section{Colaboradores}

M. F. S. O. Borges, L. M. G. Dotto, R. J. Koifman e M. A. Cunha participaram da análise e interpretação dos dados, redação do artigo e aprovação da versão final. P. T. Muniz colaborou na coordenação da pesquisa de campo, análise e interpretação dos dados e revisão crítica de conteúdo.

\section{Agradecimentos}

A realização desta investigação foi viabilizada por meio da colaboração acadêmica entre o Programa de Mestrado em Saúde Coletiva da Universidade Federal do Acre e o Programa de Pós-graduação Saúde Pública e Meio Ambiente da Fundação Oswaldo Cruz, a qual vem sendo apoiada com recursos do Conselho Nacional de Desenvolvimento Científico e Tecnológico (CNPq; Edital Casadinho UFAC-FIOCRUZ, processo ${ }^{\circ}$. 620024/2008-9), da Coordenação de Aperfeiçoamento de Pessoal de Nível Superior (CAPES; programas PROCAD-NF 1442/2007 e PROCAD-NF 2557/2008) e Ministério da Saúde.

\section{Referências}

1. Instituto Nacional de Câncer. Estimativa 2010: incidência de câncer no Brasil. Rio de Janeiro: Instituto Nacional de Câncer; 2009.

2. World Health Organization/International Agency for Research on Cancer. Cervix cancer screening. Lyon: IARC Press; 2005. (IARC Handbooks of Cancer Prevention, 10).

3. Caetano R, Vianna CMM, Thuler LCS, Girianelli VL. Custo-efetividade no diagnóstico precoce do câncer de colo uterino no Brasil. Physis (Rio J.) 2006; 16:99-118.

4. Day NE. The epidemiological basis for evaluation of different screening policies. In: Hakama M, Miller AB, Day NE, editors. Screening for cancer of the uterine cervix. Lyon: International Agency for Research on Cancer; 1986. p. 149-60.

5. World Health Organization. National cancer control programmes: policies and managerial guidelines. 2nd Ed. Geneva: World Health Organization; 2002.

6. Departamento de Atenção Básica, Secretaria de Atenção à Saúde, Ministério da Saúde. Controle dos cânceres do colo do útero e da mama. Brasília: Ministério da Saúde; 2006.
7. Muller DK, Dias-da-Costa JS, Luz AMH, Olinto MTA. Cobertura do exame citopatológico do colo do útero na cidade de São Leopoldo, Rio Grande do Sul, Brasil. Cad Saúde Pública 2008; 24:2511-20.

8. Pinho AA, França-Junior I. Prevenção do câncer de colo do útero: um modelo teórico para analisar o acesso e a utilização do teste de Papanicolaou. Rev Bras Saúde Matern Infant 2003; 3:95-112.

9. Instituto Nacional de Câncer. Inquérito domiciliar sobre comportamentos de risco e morbidade referida de doenças e agravos não transmissíveis: Brasil, 15 capitais e Distrito Federal, 2002-2003. Rio de Janeiro: Instituto Nacional de Câncer; 2004.

10. Figueiredo ICR, Jaime PC, Monteiro CA. Fatores associados ao consumo de frutas, legumes e verduras em adultos da cidade de São Paulo. Rev Saúde Pública 2008; 42:777-85.

11. World Health Organization. Global recommendations on physical activity for health. Geneva: World Health Organization; 2010.

12. Martins LFL, Thuler LCS, Valente JG. Cobertura do exame de Papanicolau no Brasil e seus fatores determinantes: uma revisão sistemática da literatura. Rev Bras Ginecol Obstet 2005; 27:485-92. 
13. Pinho AA, França-Júnior I, Schraiber LB, D’Oliveira AFPL. Cobertura e motivos para a realização ou não do teste de Papanicolaou no município de São Paulo. Cad Saúde Pública 2003; 19 Suppl 2: S303-13.

14. Quadros CAT, Victora CG, Dias-da-Costa JS. Coverage and focus of a cervical cancer prevention program in southern Brazil. Rev Panam Salud Pública 2004; 16:223-32.

15. Silva DW, Andrade SM, Soares DA, Tutini B, Schneck CA, Lopes ML. Cobertura e fatores associados com a realização do exame Papanicolaou em município do Sul do Brasil. Rev Bras Ginecol Obstet 2006; 28:24-31.

16. Oliveira MMHN, Silva AAM, Brito LMO, Coimbra LC. Cobertura e fatores associados à não realização do exame preventivo de Papanicolaou em São Luís, Maranhão. Rev Bras Epidemiol 2006; 9: 325-34.

17. Szwarcwald CL, Viacava F, Vasconcellos MTL, Leal MC, Azevedo LO, Queiroz RSB, et al. Pesquisa mundial de saúde - 2003: o Brasil em números. RADIS Comunicação em Saúde 2004; 1:14-33.

18. Instituto Brasileiro de Geografia e Estatística. Pesquisa Nacional por Amostra de Domicílios: acesso e utilização dos serviços de saúde (PNAD 2003). Rio de Janeiro: Instituto Brasileiro de Geografia e Estatística; 2003.

19. Instituto Brasileiro de Geografia e Estatística. Pesquisa Nacional por Amostra de Domicílios: um panorama da saúde no Brasil (PNAD 2008). Rio de Janeiro: Instituto Brasileiro de Geografia e Estatística; 2010.

20. World Health Organization. Cytological screening in the control of cervical cancer: technical guidelines. Geneva: World Health Organization; 1988.

21. Etlinger D, Pereira SMM, Oikawa KF, Marin AC, Araujo RS, Souza CJ, et al. Campanha de prevenção de câncer cervical: estudos no Instituto Adolfo Lutz mostram a necessidade de avaliação na faixa etária. Rev Inst Adolfo Lutz 2008; 67:64-8.

22. Leal, EAS, Leal Junior OS, Guimarães MH, Vitoriano MN, Nascimento TL, Costa OLN. Lesões precursoras do câncer de colo em mulheres adolescentes e adultas jovens do município de Rio Branco - Acre. Rev Bras Ginecol Obstet 2003; 25:81-6.

23. American Cancer Society. Global cancer facts \& figures. 2nd Ed. Atlanta: American Cancer Society; 2011.

24. Stival CO, Lazzarotto M, Rodrigues YB, Vargas VRA. Avaliação comparativa da citopatologia positiva, colposcopia e histopatologia: destacando a citopatologia como método de rastreamento do câncer do colo do útero. Rev Bras Anal Clín 2005; 37:215-8.
25. Parkin DM, Bray F, Ferlay J, Pisani P. Global cancer statistics, 2002. CA Cancer J Clin 2005; 55:74-108.

26. Zeferino LC, Pinotti JA, Jorge JPN, Westin MCA, Tambascia JK, Montemor EBL. Organization of cervical cancer screening in Campinas and surrounding region, São Paulo State, Brazil. Cad Saúde Pública 2006; 22:1909-14.

27. Carlotto K, Cesar JA, Hackenhaar AA, Ribeiro PRP. Características reprodutivas e utilização de serviços preventivos em saúde por mulheres em idade fértil: resultados de dois estudos transversais de base populacional no extremo Sul do Brasil. Cad Saúde Pública 2008; 24:2054-62.

28. Albuquerque KM, Frias PG, Andrade CLT, Aquino EMC, Menezes G, Szwarcwald CL. Cobertura do teste de Papanicolaou e fatores associados à nãorealização: um olhar sobre o Programa de Prevenção do Câncer do Colo do Útero em Pernambuco, Brasil. Cad Saúde Pública 2009; 25 Suppl 2:S301-9.

29. Koval AE, Riganti AA, Foley KL. CAPRELA (Cancer Prevention for Latinas): findings of a pilot study in Winston-Salem, Forsyth County. N C Med J 2006; 67:9-16.

30. McPhee SJ, Nguyen TT. Cancer, cancer risk factors, and community based cancer control trials in Vietnamese Americans. Asian Am Pac Isl J Health 2000; 8:18-31.

31. Martins LFL. Fatores associados à não realização do exame de Papanicolaou: estudo transversal de base populacional em duas capitais brasileiras. Rev Bras Cancerol 2006; 52:197.

32. Gasperin SI, Boing AF, Kupek E. Cobertura e fatores associados à realização do exame de detecção do câncer de colo de útero em área urbana no Sul do Brasil: estudo de base populacional. Cad Saúde Pública 2011; 27:1312-22.

33. Amorim VMSL, Barros MBA, César CLG, Carandina $\mathrm{L}$, Goldbaum M. Fatores associados à não realização do exame de Papanicolaou: um estudo de base populacional no Município de Campinas, São Paulo, Brasil. Cad Saúde Pública 2006; 22:2329-38.

34. Barros MBA. Inquéritos domiciliares de saúde: potencialidades e desafios. Rev Bras Epidemiol 2008; 11:6-19.

Recebido em 04/Out/2011

Versão final reapresentada em 02/Fev/2012

Aprovado em 08/Fev/2012 